\title{
Overall parameters for the characterization of non-uniformly totally polarized beams
}

\author{
R. Martínez-Herrero, P.M. Mejías *, G. Piquero \\ Departamento de Óptica, Facultad de Ciencias Físicas, Universidad Complutense de Madrid, 28040 Madrid, Spain
}

Received 4 January 2006; received in revised form 21 February 2006; accepted 22 February 2006

\begin{abstract}
Several overall parameters are introduced to characterize the linear or circular polarization content of a non-uniformly totally polarized beam over the region of its wavefront where the irradiance is significant. These figures of merit are determined from the values of the Stokes parameters. The physical meaning of the proposed parameters is tested by computing some numerical examples, and their measurability is checked by considering non-uniformly totally polarized fields generated after propagation through uniaxial anisotropic materials.
\end{abstract}

(C) 2006 Elsevier B.V. All rights reserved.

PACS: 41.85.Ew; 42.25.Ja

Keywords: Beam characterization; Beam polarization; Polarization standards

\section{Introduction}

As is well known, the standard degree of polarization $P$ of a general field is defined in terms of the Stokes parameters [1]. Light beams could then be classified as totally $(P=1)$, partially $(0<P<1)$ or non-polarized $(P=0)$ fields. The experimental method usually applied to determine $P$ involves measurements of the integrated beam irradiance over the full detection area for different orientations of a polarizer and a quarter-wave plate. But this procedure only makes sense if the beam is assumed to have uniform polarization properties over its cross-sectional area. In other words, it can be used when uniformly polarized fields are considered. However, beams exhibit, in general, spatially distributed polarization states [2-21]. For this kind of fields, the overall parameter $P$ cannot properly characterize their polarization properties.

It would then be useful to handle meaningful overall measurable parameters that provide information, in a

\footnotetext{
* Corresponding author. Tel./fax: +34 913944673.

E-mail address: pmmejias@fis.ucm.es (P.M. Mejías).
}

simple way, about the linear or circular polarization content of a non-uniformly totally polarized beam over the regions of its wavefront where the irradiance is not negligible. This is the aim of the present work. The parameters we will define in the paper could be considered, in a sense, as complementary to the so-called weighted degree of polarization, introduced some years ago [11].

The paper is arranged as follows: In the following section, the formalism and some preliminary definitions are given. In Section 3, the proposed characteristic parameters are introduced. In order to test their physical meaning, some numerical example are computed in Section 4. In the same section, their measurability is checked by generating non-uniformly totally polarized beams at the output of uniaxial anisotropic materials. Finally, the main conclusions are summarized in Section 5.

\section{Formalism and definitions}

Let us consider quasi-monochromatic optical fields. For uniformly polarized beams, it is well known that the degree of polarization $P$ is defined as follows [1]: 
$P=\sqrt{\frac{s_{1}^{2}+s_{2}^{2}+s_{3}^{2}}{s_{0}^{2}}}$,

where $s_{i}, i=0,1,2,3$, are the Stokes parameters measured at some plane transverse to the direction of propagation $z$. Since the field has been assumed to be uniformly polarized, the Stokes parameters do not depend on the point $(x, y)$ across the beam profile, and are proportional to the value obtained after integration throughout the wavefront. Consequently, to measure them we can handle either a photodetector or a CCD camera placed at the observation plane. Using a polarizer and a quarter-wave plate, one can write the well-known expressions

$s_{0}=I_{0}+I_{90}=I_{45, \lambda / 4}+I_{135, \lambda / 4}$,

$s_{1}=I_{0}-I_{90}$,

$s_{2}=I_{45}-I_{135}$,

$s_{3}=I_{45, \lambda / 4}-I_{135, \lambda / 4}$.

In Eqs. (2.a)-(2.d) the subscripts indicate the angle that the transmission axis of the polarizer makes with the $x$-axis. To obtain $s_{3}$ the irradiance should be measured after the beam propagates successively through the quarter-wave plate (whose fast axis makes an angle $0^{\circ}$ with the $x$-axis) and the polarizer oriented so as to transmit the component in the azimuths $45^{\circ}$ and $135^{\circ}$.

Let us now consider non-uniformly polarized beams. In such a case, the Stokes parameters depend on the transverse vector $\boldsymbol{r}=(x, y)$. Accordingly, the degree of polarization becomes a spatial function and should be written in the form

$P(\boldsymbol{r})=\sqrt{\frac{s_{1}^{2}(\boldsymbol{r})+s_{2}^{2}(\boldsymbol{r})+s_{3}^{2}(\boldsymbol{r})}{s_{0}^{2}(\boldsymbol{r})}}$.

In this case, a major problem arises because, for nonuniformly polarized fields, we cannot get the standard degree of polarization $P$, given by Eq. (1), from measurements of irradiance (see Eqs. (2)) integrated throughout the transverse beam profile.

Alternative parameters would then be of interest to describe the overall spatial distribution of the polarization state over the beam cross-section. With this aim, the socalled weighted degree of polarization, $\widetilde{P}$, was introduced [11]

$\widetilde{P}=\frac{\int I(\boldsymbol{r}) P(\boldsymbol{r}) \mathrm{d} \boldsymbol{r}}{\int I(\boldsymbol{r}) \mathrm{d} \boldsymbol{r}}$,

where $P(\boldsymbol{r})$ is given by Eq. (3) and $I(\boldsymbol{r})$ is the irradiance of the light beam at each point of the observation plane. This parameter globally characterizes the polarization state over the region of the beam profile where the irradiance is significant. For example, if we consider radially totally polarized fields $[2,3,15,18-21]$, one gets $\widetilde{P}=1$, which means that this kind of fields are totally polarized throughout the transverse section, as it should be expected. However, in this case, the standard parameter $P$ equals zero, which is a meaningless result (note that, for uniformly polarized beams, $P=0$ would imply that the field is unpolarized).

In the present work, the attention will be focused on the global characterization of the so-called linear or circular polarization content at the transverse profile of nonuniformly totally polarized (NUTP) beams. This subject will be analytically introduced in the following section.

\section{Overall parameters}

From now on let us consider NUTP beams. The electric field vector, $\boldsymbol{E}(\boldsymbol{r})$, can then be represented by means of the Jones vector

$\boldsymbol{E}(\boldsymbol{r})=\left(\begin{array}{c}E_{\mathrm{s}}(\boldsymbol{r}) \\ E_{\mathrm{p}}(\boldsymbol{r})\end{array}\right)$,

where $E_{\mathrm{s}}$ and $E_{\mathrm{p}}$ denote the transverse field components orthogonal to the $z$-axis. Let us now introduce two orthogonal unitary vectors, $\boldsymbol{u}_{\mathrm{r}}=\frac{1}{\sqrt{2}}\left(\begin{array}{l}1 \\ \mathrm{i}\end{array}\right)$ and $\boldsymbol{u}_{l}=\frac{1}{\sqrt{2}}\left(\begin{array}{l}1 \\ -\mathrm{i}\end{array}\right)$, where the subscripts ' $r$ ' and ' 1 ' refer to right-handed and left-handed circularly polarized fields, respectively. Taking this into account, the vector $\boldsymbol{E}_{\mathrm{r}}=\left(\boldsymbol{E} \cdot \mathbf{u}_{\mathrm{r}}\right) \mathbf{u}_{\boldsymbol{r}}$ would represent the field at the output of an optical device that only transmits the right-handed circularly polarized component of the input field (the dot symbolizes the inner product). The magnitude of $\boldsymbol{E}_{\mathrm{r}}$ could then be evaluated in two equivalent ways:

(a) Let us define

$\left\|\boldsymbol{E}_{\mathrm{r}}\right\|^{2} \equiv \int\left|\boldsymbol{E}_{\mathrm{r}}(\boldsymbol{r})\right|^{2} \mathrm{~d} \boldsymbol{r}$,

where the integration extends throughout the beam crosssection. This expression can be understood as the beam power associated to the "right-handed circular content" (RCC) $\boldsymbol{E}_{\mathrm{r}}$ of the field. In addition, it follows at once:

$\left\|\boldsymbol{E}_{\mathrm{r}}\right\|^{2}=\frac{1}{2} \int s_{0}(\boldsymbol{r}) \mathrm{d} \boldsymbol{r}+\frac{1}{2} \int s_{3}(\boldsymbol{r}) \mathrm{d} \boldsymbol{r}$.

The total power would read

$\|\boldsymbol{E}\|^{2} \equiv \int\left[\left|\boldsymbol{E}_{s}(\boldsymbol{r})\right|^{2}+\left|\boldsymbol{E}_{p}(\boldsymbol{r})\right|^{2}\right] \mathrm{d} \boldsymbol{r}=\int s_{0}(\boldsymbol{r}) \mathrm{d} \boldsymbol{r}$,

and we have

$\frac{\left\|\boldsymbol{E}_{\mathrm{r}}\right\|^{2}}{\|\boldsymbol{E}\|^{2}}=\frac{1}{2}+\frac{1}{2} \frac{\int s_{3}(\boldsymbol{r}) \mathrm{d} \boldsymbol{r}}{\int s_{0}(\boldsymbol{r}) \mathrm{d} \boldsymbol{r}}$.

This equation would then provide the ratio between the power associated to the RCC of the beam and its total power.

(b) Let us now consider the percentage of the beam irradiance transmitted through a right-handed circular polarizer. This ratio, $\alpha$, can formally be expressed in the form

$\alpha(\boldsymbol{r})=\frac{\left|\boldsymbol{E} \cdot \boldsymbol{u}_{\mathrm{r}}\right|^{2}}{|\boldsymbol{E}|^{2}}=\frac{s_{0}(\boldsymbol{r})+s_{3}(\boldsymbol{r})}{2 s_{0}(\boldsymbol{r})}$. 
Averaging this value over the region of the beam profile where the irradiance is significant gives

$\tilde{\alpha}=\frac{\int \alpha(\boldsymbol{r}) s_{0}(\boldsymbol{r}) \mathrm{d} \boldsymbol{r}}{\int s_{0}(\boldsymbol{r}) \mathrm{d} \boldsymbol{r}}$,

and we readily find

$\tilde{\alpha}=\frac{\left\|\boldsymbol{E}_{\mathrm{r}}\right\|^{2}}{\|\boldsymbol{E}\|^{2}}$,

which is the result we are looking for. This expression shows that, in order to evaluate the magnitude of $\boldsymbol{E}_{\mathrm{r}}$, both points of view, (a) and (b), are equivalent.

Since the factors $1 / 2$ inside Eq. (9) are rather cumbersome in the calculations, we introduce the following parameters

$\tilde{\rho}=\frac{\int \rho(\boldsymbol{r}) s_{0}(\boldsymbol{r}) \mathrm{d} \boldsymbol{r}}{\int s_{0}(\boldsymbol{r}) \mathrm{d} \boldsymbol{r}}$

and

$\sigma_{\rho}=\frac{\int(\rho(\boldsymbol{r})-\tilde{\rho})^{2} s_{0}(\boldsymbol{r}) \mathrm{d} \boldsymbol{r}}{\int s_{0}(\boldsymbol{r}) \mathrm{d} \boldsymbol{r}}$,

where

$\rho(\boldsymbol{r}) \equiv \frac{s_{3}(\boldsymbol{r})}{s_{0}(\boldsymbol{r})}$.

Note that $\tilde{\alpha}=\frac{1}{2}+\frac{1}{2} \tilde{\rho}$. In other words, $\tilde{\rho}$ and $\tilde{\alpha}$ have the same physical meaning. The parameter $\tilde{\rho}$ computes mainly in those regions where the irradiance is not negligible and ranges from -1 (pure left-handed circularly polarized light) to +1 (pure right-handed circularly polarized light). The value $\tilde{\rho}=0$ corresponds to a pure (in general, nonuniformly) linearly polarized field. As it is apparent from the definition, $\tilde{\rho}$ can be obtained in a simple way by measuring the Stokes parameters $s_{0}$ and $s_{3}$ integrated over the full detection area, even though the beam is nonuniformly polarized.

The parameter $\sigma_{\rho}$ gives the dispersion of the values of $\rho(\boldsymbol{r})$ across the beam section. In fact, it represents the variance of $\rho(\boldsymbol{r})$ (the local value of the Stokes parameter $s_{0}(\boldsymbol{r})$ behaving as a density function). Therefore, this parameter globally characterizes the uniformity of the ratio $\rho(\boldsymbol{r})$ over the beam profile. In particular, when $\tilde{\rho}=0=\sigma_{\rho}$, it can be concluded that the beam is linearly polarized throughout its transverse section.

\section{Application to several examples}

To proceed further into the physical meaning of this parameter, let us now consider a non-uniformly totally polarized beam whose electric field vector $\boldsymbol{E}$ at the plane $z=0$ is given by the Jones vector

$\boldsymbol{E}(r)=E_{0} \exp \left(-\frac{r^{2}}{\omega_{0}^{2}}\right)\left(\begin{array}{c}1 \\ \exp (-\mathrm{i} g(r))\end{array}\right)$

with $g(r)=\frac{\pi}{2} \exp \left[-(b r)^{n}\right], \quad n=4$,

where $E_{0}$ is an amplitude factor, $r$ denotes the radial polar coordinate, and $\omega_{0}$ and $b$ are constants $\left(\omega_{0}\right.$ represents the beam size at the waist plane and $b^{-1}$ is a measure of the width of the function $g(r)$ ). Note that the whole beam exhibits a Gaussian intensity profile and the field shows an abrupt transition from a circularly polarized state near the beam center to a $45^{\circ}$-azimuth linearly polarized state outside the central region. This is illustrated in Fig. 1(a), where the ratio $\operatorname{Im}\left(E_{\mathrm{s}}(r) / E_{\mathrm{p}}(r)\right)$ has been plotted for $b=1 \mathrm{~mm}^{-1}$. A representation of the change from circular to linear polarization is sketched in Fig. 1(b).

The parameters $\tilde{\rho}$ and $\sigma_{\rho}$ have been computed in terms of the ratio $\omega_{0} / b$ (see Fig. 2). When $\omega_{0} / b \ll 1$, then $\tilde{\rho} \approx-1$ and $\sigma_{\rho} \approx 0$, so that the beam behaves as a lefthanded circularly polarized field inside the region where the irradiance takes appreciable values. For $\omega_{0} / b \gg 1$, $\tilde{\rho} \approx 0$ and the beam becomes linearly polarized over the whole beam profile except over a small region closed to its center. The intermediate case $\omega_{0} / b$ gives $\tilde{\rho}=-0.41$ and $\sigma_{\rho}=0.17$, and a mixture of both states of polarization (linear and left-handed circular) would be present.

In order to check the measurability of the proposed parameters, let us first consider the beam emitted by a $\mathrm{He}-\mathrm{Ne}$ laser source Spectra Physics 117A (intensity and frequency-stabilized mode), linearly (>1000:1) and uniformly polarized according to the manufacturer specifications. In the experiment we use a linear polarizer whose extinction ratio is $T_{1} / T_{2}=250$ and a quarter-wave plate with a transmittance $T_{3}=0.98$. Although the experimental procedure is conceptually simple, some care, however, is required because the optical components (polarization-altering devices and photodetector array) are not ideal and may

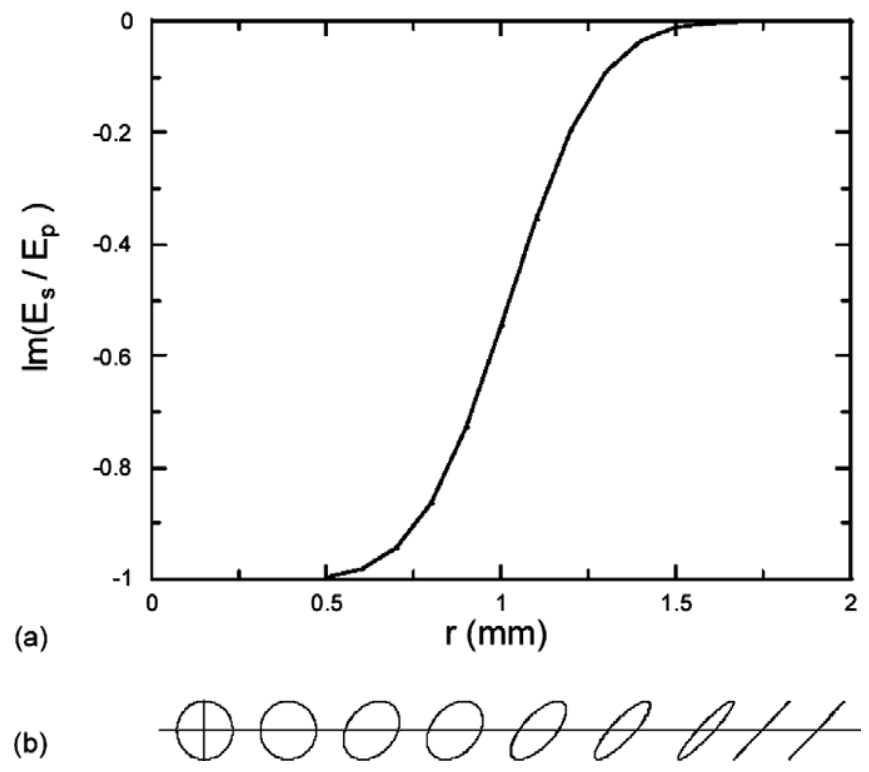

Fig. 1. (a) Ratio $\operatorname{Im}\left(E_{\mathrm{s}}(r) / E_{\mathrm{p}}(r)\right)$ versus the distance $r$ to the origin. (b) Schematic diagram of the polarization state for different $r$ in the range $[0,2] \mathrm{mm}$ from the origin. 

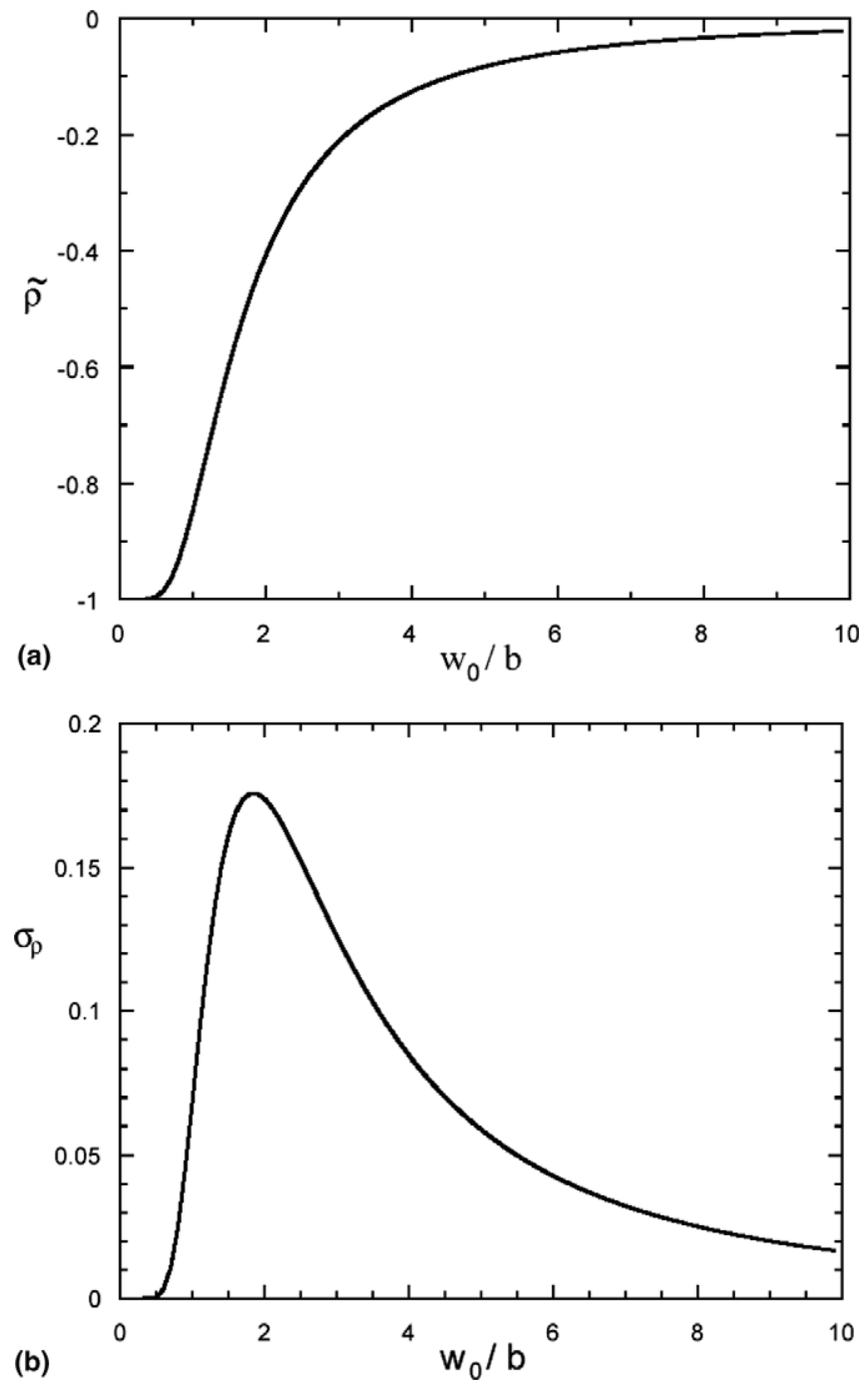

Fig. 2. Parameters $\tilde{\rho}$ and $\sigma_{\rho}$ for the beam model given by Eq. (16): (a) $\tilde{\rho}$ versus the ratio $\omega_{0} / b$; (b) $\sigma_{\rho}$ versus the ratio $\omega_{0} / b$.

exhibit certain harmful effects. Special attention should be taken to align the system in order that the beam crosses through the center of both the polarizer and the retarder plate. In such a case, when these components rotate, the influence of their spatial inhomogeneities on the global intensity will be drastically reduced. The images were taken with a CCD camera Pulnix TM-765 and a laser beam analyzer from Spiricon.

For this reference beam, it has been obtained $\tilde{\rho}=0.0043$ and $\sigma_{\rho}=0.0001$. In addition, when left- and right-handed circularly polarized beams where synthesized by means of the quarter-wave plate we got $\tilde{\rho}=-1.008, \sigma_{\rho}=0.001$ and $\tilde{\rho}=1.081, \sigma_{\rho}=0.001$, respectively. We see that all these measured values show a reasonable agreement with the manufacturers' data.

Let us now measure the parameters $\tilde{\rho}$ and $\sigma_{\rho}$ in a more involved case, namely, a non-uniformly totally polarized beam generated after propagation through uniaxial anisotropic material. In the experiments, the previously cali-

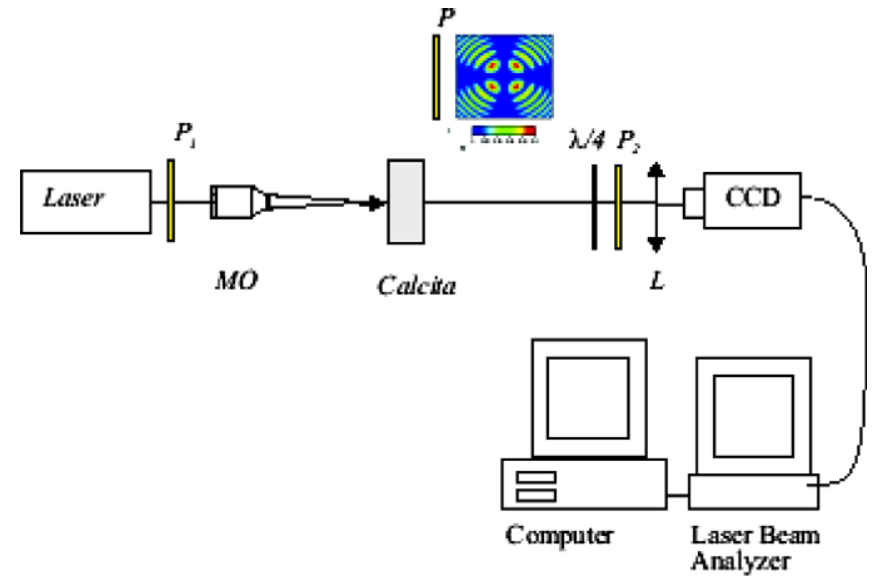

Fig. 3. Experimental set-up used to measure the parameters $\tilde{\rho}$ and $\sigma_{\rho}$ at the output of a calcite crystal: MO, microscope objective; L, convergent lens; $\mathrm{P}$, polarizer; $\lambda / 4$, quarter-wave plate.

brated $\mathrm{He}-\mathrm{Ne}$ laser beam was used. The anisotropic medium is a calcite crystal whose optic axis is oriented along the propagation direction $[14,16]$. If the incident beam is collimated and parallel to the optic axis, the material behaves as an isotropic crystal and the polarization state does not change from one point to another of the beam cross-section. In order to synthesize a NUTP beam, we have to use a slightly convergent or divergent beam impinging on the crystal [1]. This can be implemented by using a microscope objective in front of the calcite plate (see Fig. 3). On the other hand, a convergent lens was used to focus the beam into the CCD camera. The experiments gave $\widetilde{P}=1.036, \tilde{\rho}=0.044$ and $\sigma_{\rho}=0.22$. From the value of $\widetilde{P}$, it is concluded at once that the output field essentially behaves as a totally polarized beam throughout its transverse section, as expected. The value of $\tilde{\rho}$ means that the average polarization is linear over the region of the beam cross-section where the irradiance is not negligible. In addition, since $\sigma_{\rho}$ is significant, we could infer that the output beam is not uniformly polarized. Note finally that this behavior agrees with the expression of the Jones vector at the output of a calcite crystal (cf. Ref. [16]).

\section{Conclusions}

Two overall parameters, $\tilde{\rho}$ and $\sigma_{\rho}$, have been proposed to characterize the predominant state of polarization (circular or linear) over the cross-sectional area of nonuniformly totally polarized beams. This characterization refers to the regions where the beam irradiance is significant. The proposed parameters are given in terms of the Stokes parameters. In particular, $\tilde{\rho}$ can be determined by measuring $s_{0}$ and $s_{3}$ integrated throughout the beam cross-section, even though the field is non-uniformly polarized. The measurement procedure has revealed to be simple and has been checked by testing laser manufacturer's specifications and by considering non-uniformly totally polarized beams at the output of uniaxial anisotropic crystals. 


\section{Acknowledgements}

This work has been supported by the Ministerio de Educación y Ciencia of Spain, Project FIS2004-1900, and by the Universidad Complutense-Comunidad de Madrid, within the framework of the Research Groups Program 2005-06.

\section{References}

[1] M. Born, E. Wolf, Principles of Optics, seventh ed., Cambridge University Press, Cambridge, 1999.

[2] T. Endogen, D.G. Hall, J. Appl. Phys. 68 (1990) 1435.

[3] S.C. Tidwell, D.H. Ford, W.D. Kimura, Appl. Opt. 29 (1990) 2234.

[4] T. Endogen, O. King, G.W. Wicks, D.G. Hall, E. Anderson, M.J. Rooks, Appl. Phys. Lett. 60 (1992) 1921.

[5] S.C. Tidwell, G.H. Kim, W.D. Kimura, Appl. Opt. 32 (1993) 5222.

[6] Q. Lü, S. Dong, H. Weber, Opt. Quantum Electron. 27 (1995) 777.
[7] P.L. Greene, D.G. Hall, J. Opt. Soc. Am. A 13 (1996) 962.

[8] R. Martínez-Herrero, P.M. Mejías, J.M. Movilla, Opt. Lett. 22 (1997) 206.

[9] F. Gori, Opt. Lett. 23 (1998) 241.

[10] J.M. Movilla, G. Piquero, P.M. Mejías, R. Martínez-Herrero, Opt. Commun. 149 (1998) 230.

[11] G. Piquero, J.M. Movilla, P.M. Mejías, R. Martínez-Herrero, Opt. Quantum Electron. 31 (1999) 223.

[12] I. Freund, Opt. Commun. 199 (2001) 47.

[13] P.M. Mejías, R. Martínez-Herrero, G. Piquero, J.M. Movilla, Prog. Quantum Electron. 26 (2002) 65.

[14] D. Provenziani, A. Ciattoni, G. Cincotti, C. Palma, F. Ravaccia, C. Sapia, Opt. Express 10 (2002) 699

[15] R. Dorn, S. Quabis, G. Lenchs, Phys. Rev. Lett. 91 (2003) 233901.

[16] G. Piquero, J. Vargas-Balbuena, Eur. J. Phys. 25 (2004) 793.

[17] G. Volpe, D. Petrov, Opt. Commun. 237 (2004) 89.

[18] T.D. Visser, J.T. Foley, J. Opt. Soc. Am. A 22 (2005) 2527.

[19] Y. Zhang, L. Wang, C. Zheng, J. Opt. Soc. Am A 22 (2005) 2542.

[20] T. Moser, H. Glur, V. Romano, F. Pigeon, O. Parriaux, M.A. Ahmed, T. Graf, Appl. Phys. B 80 (2005) 707.

[21] S. Quabis, R. Dorn, G. Leuchs, Appl. Phys. B 81 (2005) 597. 